giste, qui montrera ce merveilleux appareil, susceptible de donner de l'énergie à aussi bon compte que bien des chutes d'eau économiques, pouvant être aussi bien considéré comme un gazogène dont la fonte est un sous-produit. Enfin, nous consacrerons quelques articles aux moteurs à huile lourde du type Diesel, qui sont bien, à ce qu'il semble, les véritables machines de secour's de l'avenir, pour de très nombreuses usines employant le moteur électrique aux confins des réscaux de distribution d'énergie, ou le moteur hydraulique sur d'inconstants petits cours d'eau.

La puissance unitaire des turbines à vapeur croît avec une rapidité surprenante, et, pour en donner une idée, en attendant les études annoncées, nous reproduisons ici une curieuse photographie: un tracteur de 32 chevaux-avoine, remorquant une partie d'une machine de 25 ooo chevauxvapeur. Celte photographic représente en effet le transport de l'une des plus grosses pièces d'une turbine à vapeur de $25000 \mathrm{HP}$, construite par la Compagnie Electro-Mécanique du Bourget, et destinée à l'usine de la Société d'Electricité de Paris, à Saint-Denis. Le rotor de la turbine (que représente la photographic) pèse 38 tonnes. La partie inférieure

\section{SYMBOLES \\ DIAGRAMMES POUR COURANTS ALTERNATIFS SPÉCIFICATIONS DES MACHINES}

Décisions de la Commission Electrotechnique Internationale (Réunion de Turin-IgI I)

Les décisions suivantes sont adoptées provisoirement par la Commission électrotechnique internationale :

\section{SYMBOLES}

$I^{\circ} a$. Les grandeurs électriques instantanées (variables dans le temps) sont représentées par des lettres minuscules;

$b$. Les grandeurs électriques efficaces ou constantes sont représentées par des lettres majuscules;

$c$. Les valeurs maxima des grandeurs électriques sont représentées par des lettres majuscules affectées de l'indice $m$;

$d$. Les grandeurs magnétiques, constantes ou variables; sont représentées par des lettres majuscules, rondes, gothiques, grasses ou type spécial;

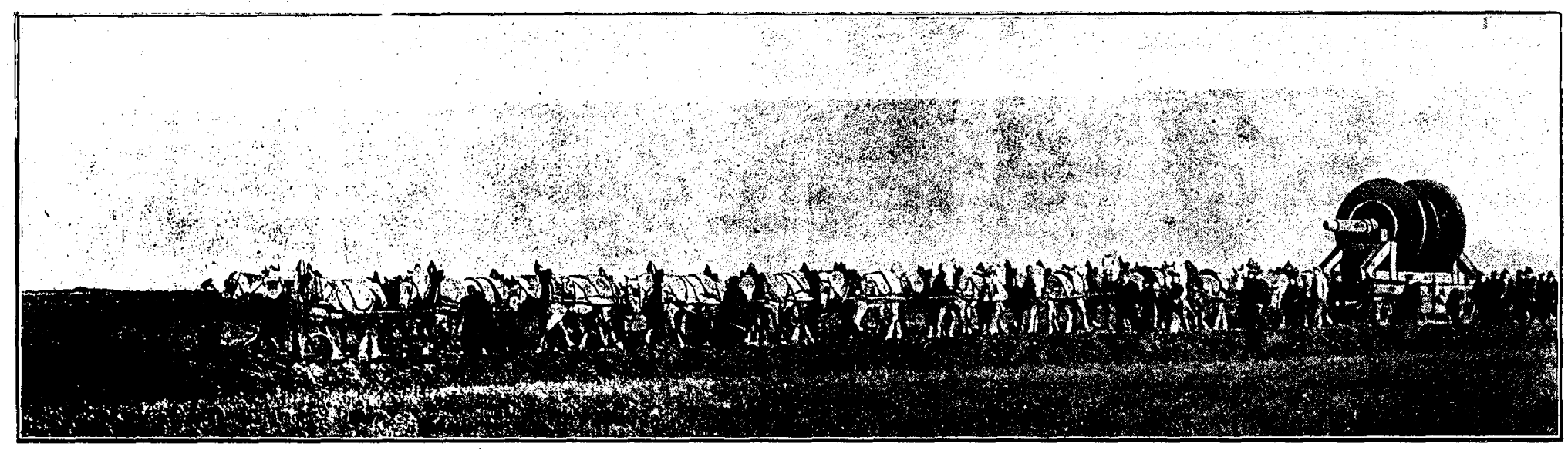

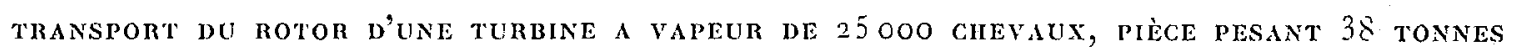

du cylindre pèse / $\mathrm{r}$ tonnes. Si l'on ajoute que chacun des charriots portant ces pièces pèse plus de r2 tonnes, on comprendra que ces transports aient nécessité des altelages de 3 . chevaux pour le premier et 35 chcvaux pour le secord. En laison de leurs dimcnsions, ces pièces indivisibles n'aki. raient pu etre transportées par chemin de fer depuis les ateliers du Bourget jusqu'à l'usine de Saint-Denis.

On sait que cette usine est actuellement la plus forte station centrale de l'Europe ; clle comporte, en effet, so turbogroupes Brown, Boveri-Patson's, de toooo IlP chacun. C'est ì ces groupes que rient s'ajouter le turbo-altemateur de 25000 IIP. Grâce aux perfectionnements apportés et à la diminution d'encombrement qu'ils ont permis de réaliser, on a pu installer cetle machine dans l'emplacement précédemment réservé à un groupe de to ooo HP. Le nouveau groupe a pour dimensions :

Largeur $=3,40$ mètres $;$ Iongueur totale $=x 3,60$ mètres (dont 8,50 pour la turbine et 5 , to pour l'alternateur). Son poids total est de 300 tonnes environ, dont 140 tonnes pour la turbine seule.

La houille blanche est battue; nlle avait des turbines de 15 ooo chevaux; c'est maintenant la houille noire qui délient le record de la puissance unilaire. La revanche est probablement possible. Mais quand l'aurons-nous?

\section{E.-F. Côtre.}

$e$. Les valeurs maxima des grandeurs magnétiques périon diques sont représentées par des lettres majuscules rondes, gothiques, grasses ou type spécial affectées de l'indice $m$;

$f$. Les grandeurs suivantes sont représentées par les lettres ci-après :

Force électromotrice ........ E, c

Quantité d'électricité .......QQ, q

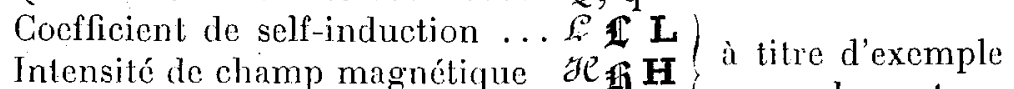
Induction magnétique $\left.\ldots \ldots \ldots \ldots \boldsymbol{B}_{\mathbf{B}} \mathbf{B}\right\}$ seulement

$$
\begin{aligned}
& \text { Longueur . ........ L, } 1 \\
& \text { Masse } \ldots \ldots \ldots \ldots \ldots \ldots \text { M, m } \\
& \text { Temps.......... T, } \mathbf{t}
\end{aligned}
$$

$2^{\circ}$ Les lettres I, E, R, sont adoptées définitivement pour représenter respectivement le courant, la force électromotrice ct la résistance, dans l'expression algébrique de la loi d'Ohm.

$3^{\circ}$ Dans les questions relatives aux courants alternatifs, l'expression Puissance réaclive est adoptée pour désigner la quantité UI $\sin \varphi$.

\section{DIAGRAMME POUR GOURANTS ALTERNATIFS}

Dans les représentations graphiques des grandeurs électriques ou magnétiques alternatives, l'angle correspondant 
à unc avance de phase doit être porté dans le sens inverse du mouvement des aiguilles d'une montre.

Nole. - L'expression symbolique de l'impédance d'une E I bobine de réaction ayant une résistance $\mathrm{R}$ et un

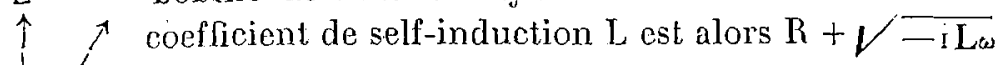
$\int$ et celle d'un condensateur de capacité $\mathrm{C}, \frac{1}{\sqrt{-\mathrm{I} \mathrm{C}_{\omega}}}$
$(\omega=2 \pi \times$ fréquence).

La convention dont il s'agit conduit à la figure cicontre dans le cas d'un courant of en retard de phase sur unc force élpetromotrice OF.

\section{SPEGIFICATIONS DES MACHINES}

En ce qui concerne la puissance des machines à courant conlinu el lorsqu'il n'en est spécifé autrement:

a. Les générateurs électriques sont caractérisés par la puissance électrique disponible à leurs bornes ;

b. Les moleurs électriques sont caractérisés par la puissanco mécanique disponible sur leur arbre.

c. Les puissances électrique el mécanique sont exprimées an walts internationaux.

\section{ÉLEGTROMÉTALLURGIE}

\author{
LE FOUR ÉLECTRIQUE \\ Appliqué à la production directe de la fonte et à l'obtention \\ de l'acier doux en partant du minerai
}

Les fragmients de ferro-silicium trouvés dès 1898 dans les brasquages des fours à carbure de calcium, qui ne pouvaient provenir que de la silice de la chaux et des cendres de l'anthrncite, de la pyrite que contenait celui-ci et du fer des ringards qui fondaient quand on piquait la charge pour dégager l'électrode suspendue et faire descendre le mélange, montraient que l'on pouvait, malgré la température de l'arc, éviter la volatilisation du fer dans le four électrique. A cette époque (1898), M. Stassano essayait, sans succès, de réduire lo minerai de fer. En rgor, M. Harmet présentait son projet de haut-hourneau électrique. Un peu plus tard, le gouverncment du Canada, désireux d'utiliser à la fois la puissance des rivières de celte contréc et les gisements inépuisables to mincrai de fer qu'elle possède faisait procéder, par une commission compétente, à des essais de réduction, dans les fours connus ì ce moment.

Ces essais eurent comme conséquence l'installation d'un form d'Hléroult à Sault-Sainte-Marie, au Canada, ct excitèrent les recherches dans celte voie; il ne fut, toutefois, pas publié gr'and'chose sur leurs résultats et ce n'est que par la communication faite par MM. J.-A. Lefreer el E. Onelberc au Jern. Kontoret (Associalion Suédoise des Maâtres de Forges), Je 3r mai $19 x t$, que l'on a connu les résultats d'un essai prolongé fait avec un matériel approprié en vue d'arriver à la réduction directe du minerai de fer au four élcctrique.

Nous nous proposons, dans les pages qui vont suivre, de présenter au lectcur de La Houille Blanche les diverses communicalions faites sur l'expérience poursuivic à Trollhällan. les dimensions du four employé, les conditions d'installation de force, l'aménagement des moyens de transport du minerai et du combustible, tout concourt à faire des expćriences poursuivies à Trollhättan une démonstration de ce que l'on peut actuellement obtenir du four électrique et les renseignements que l'on a sur elles ainsi que les déductions qu'on en peut tirer permeltront d'apprécier bien plus aisćment les efforts poursuivis pour faire de la sidérurgic électrique une industrie autonome el de se former une opinion sur les chances de son avenir comme telle.

Nous ferons de nombreux emprunts à "Metallurgical and Chemical Engineering", à "Stahl und Eisen", "Enginecring", "The Iron \& Coal Review " et "La Revue de Mélallurgie ", notre excellent confrère aussi intércssant pour l'administrateur d'usine que pour le technicien, nous les prions ici d'accepter nos remerciements.

A la séance de l' "Iron \& Steel Institute " de mai rgog, M. E.-J. Luungberg, président de la "Stora Kopparberg Bergslag's A. B. » (Suède), à laquelle apparticnt l'usine de Domnarfvet, fit une communication sur l'aptitude du four électrique de MM. Grönwall, Lindblad et Stalhane, à la réduction directe du minerai de fer. Plus tard, M. Lars Yngström, directcur de la Société, publia un rapport complet sur les expéricnces faites el démontra qu'on pouvait baser une industrie sur l'emploi de ce four.

En Suède, où le four avait pris naissance, on reconnut promptement les mérites du procédé, d'autant que les conditions naturelles y sont très favorables à l'électrosidérurgie. Riche minerai de fer abondant, force motrice hydraulique bon marché, pénurie de houille et, par suite, de coke. I.es hauls-fourneaux y sont alimentés de charbon de bois dont le prix augmente d'année en année. La possibilité de remplacer environ les deux liers du combustible par l'énergie électrique, en mêne temps que lat perspective d'amóliorer encore la qualité du fer de Suède déjà universellement, appréciée, a donné au succès de ce procédé unc importance nationale et c'est ainsi qu'on l'a considéré.

Il existe en Suède une Association vieille de plus de cent cinquante ans, appelée "Jern Kontoret", qui groupe tous les mâtres de forges du pays et joue mu grand rôle dans l'industrie sidérurgique suédoise, elle a pour objet de donner une assistance technique et financiere à ses membres et, d'une façon générale, de perfectionnel el développer l'industrie sidérurgique du pays.

Après avoir soigneusement analysć les résultalts des expériences de Domnarfvet, le comité du "Jern Kontorel " décida de contracter un accord avec l' "Electromolall $\Lambda$. B. ", constituée par les inventeurs du four et de paire les frais d'une installation destince à éludier is fond la valeur industrielle du procédé. Le fail que c'élait lat première fois que cetle association s'engilgeail dans une entreprise industrielle: pour son propre compte, prouve l'inportance fu'clle allatchait au procédé. Le gouvernement suédois offrit aussi de contribuer ì l'entreprise en fommissant ì son usime de T'rolJhättan l'énergie électrique ì un prix mominal.

L'installation a commeneć à fonctionner lo lis novembre

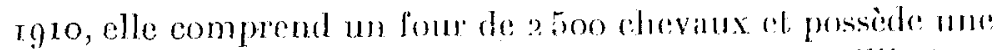
organisalion profondémont éludié (n) vue de recuesillir tons les renseignements scientiligues et industricls désirables.

Entre temps, l'industrie frivée ne reslial pas inaclive. A la fin des essais poursuivis par Jes inventeurs, te four des Forges de Domnarfel fut repris par la "Stora Kopparbergs Bergslag's A. B. ", il qui il appartenait, qui continuat a de faire marcher pour ses propres essits. Jille construit, acluellement un nouveau four de hooo chevaux, premice. d'une série de fo ooo chevaux. l'usine projelé aura une caipacité annuclle de 120000 lonnes de fonte at coutstra près de 18 millions de franes. 